\title{
Ronald Melzack and Patrick Wall. La teoría de la compuerta. Más allá del concepto científico dos universos científicos dedicados al entendimiento del dolor
}

\author{
J. C. Acevedo González
}

Unidad de Neurocirugía. Hospital Universitario de San Ignacio. Pontificia Universidad Javeriana. Departamento de Cirugía. Sección de Neurocirugía. Fundación Santa Fe de Bogotá. Clínica de Dolor. Instituto de Rehabilitación Física y Electrodiagnóstico. Bogotá. Colombia

Acevedo González JC. Ronald Melzack and Patrick Wall. La teoría de la compuerta. Más allá del concepto científico dos universos científicos dedicados al entendimiento del dolor. Rev Soc Esp Dolor 2013; 20(4): 191-202. ible process that leads to scientific development. The analysis of what this paper has symbolized to pain science must be done not only from a purely scientific view point but from a human one as well.

Key words: pain. Gate control. Ronald Melzack. Patrick Wall.

\section{RESUMEN}

La evolución histórica para el conocimiento del dolor ha tenido momentos cruciales en los que el curso evolutivo se modificó. Esos momentos son situaciones relevantes que cambiaron los conceptos previamente aceptados y abrieron nuevos horizontes de investigación, de entendimiento y de tratamiento. Cuando Melzack y Wall desarrollaron la "Teoría de la Compuerta" lograron aclarar fenómenos básicos del entendimiento del dolor pero igualmente abrieron múltiples tópicos de futuras investigaciones básicas y clínicas. Dieron sustento, con su teoría, a múltiples tratamientos del dolor que son la base de procedimientos que en la actualidad realizamos. Los aspectos psicológicos, el componente clínico y la base estructural anatomofisiológica fueron poco a poco conformando una de las teorías más completas de la ciencia médica y por supuesto principio básico para el mejor tratamiento del dolor. Son 50 años que se cumplen de la publicación de aquel artículo en la revista Science (Pain Mechanism: a new theory. A gate control system modulates sensory input from the skin before it evoques pain perception and response. Ronald Melzack and Patrick Wall. Science. 19 november 1965, volumen 150, number 3699), el cual ha sido reconocido hasta la actualidad como la referencia bibliográfica más utilizada en la ciencia médica en los últimos 50 años. Este artículo se escribió en un contexto científico particular y a la luz de dos vidas diferentes que ilustran un proceso ejemplar de desarrollo de la ciencia. El análisis es no solo científico sino humano 
de lo que ha sido este artículo para el entendimiento de las ciencias del dolor.

Palabras clave: Dolor. Teoría de la compuerta. Ronald Melzack. Patrick Wall.

\section{INTRODUCCIÓN}

Hace 46 años se abrió al mundo científico la "compuer$t a$ " al entendimiento de muchos de los principios básicos que explican la presencia del dolor. Dos importantes científicos desarrollaron a partir de conocimientos previos la "Teoría de la Compuerta" ("Gate Control”). Ellos publicaron en la revista Science el artículo "Pain Mechanism: a new theory". Este fue el primero de varios trabajos que desarrollaron en toda su extensión la importante teoría reconocida como base de gran parte del entendimiento de los mecanismos periféricos y centrales, en el sistema nervioso, que participan en la generación y el mantenimiento del dolor. Permitió entender que las diferentes formas de sensibilidad interactúan a nivel medular para modular el estímulo doloroso y en ocasiones controlarlo. Describe sistemas de neuronas medulares con patrones de funcionamiento opuesto que ponen en competencia mecanismos de Inhibición y de excitación, dependientes de ese primer nivel de modulación segmentaria del dolor, localizado en la médula y denominado por Ronald Melzack y Patrick Wall la "Compuerta” (1-7).

Hay momentos en la historia que no se pueden olvidar y su recuerdo nos permite enriquecernos con el proceso analítico que tuvieron los grandes científicos para llegar a concretar teorías básicas para la práctica diaria de la medicina. Esta "Teoría de la Compuerta" significó el punto de cambio en el tratamiento del dolor. No solo por su importancia neurofisiológica sino por los hechos que de ella se desencadenan de forma directa e indirecta y que permitieron mejorar el tratamiento del dolor. Aprovechamos ese recuerdo histórico para revisar la biografía de dos grandes científicos y desmenuzar ese artículo famoso, integrándolo en la realidad actual de una subespecialidad joven como es el manejo del dolor.

\section{PATRICK DAVID WALL (1925-2001)}

Nace el 25 de Abril de 1925 en la ciudad de inglesa de Nothingham, capital de la provincia de Nottinghamshire en la parte central del Reino Unido. Esta ciudad nos recuerda las historias clásicas de la Edad Media inglesa en la que los bosques de Sherwood (al norte de Nothigham) escondían al defensor de los pobres Robin Hood. El Dr. Wall nace en un hogar culto y puritano en donde su padre Thomas
Wall, una persona extrovertida, se desempeñaba inicialmente como profesor y luego trasladado para ser director general en la Universidad de Middlesex. Su madre, Ruth Cresswell, cuidaba de los niños bajo los principios puritanos ingleses. Su infancia se desarrolló bajo la comodidad de un hogar con solvencia económica y bajo la pasión de su hermano mayor quien adoraba los automóviles y los aeroplanos. El carácter de Patrick comenzó a desarrollarse desde los primeros años y su espíritu cuestionador de la autoridad se reafirmó desde muy temprana edad cuando a los 8 años un profesor le dijo que la región de Lancashire era conocida por producir gran cantidad de algodón. Investigó dicha afirmación y logró encontrar que efectivamente en dicha región se hilaba y tejía gran cantidad de algodón, pero que toda la materia prima llegaba de otras ciudades. A pesar de ser algo muy simple y sin mayor trascendencia la decepción frente a la autoridad fue mayor siendo recordada por el Dr. Wall como "el inicio de una vida de duda frente a los pronunciamientos de la autoridad”. Ese pequeño suceso marcó muchas decisiones en su actividad profesional, rechazando durante muchos años el esquema clásico de “Jefe de Departamento Médico", considerando que era una posición social cuyos patrones de funcionamiento eran deplorables marcados esencialmente por un claro abuso de autoridad. A los 10 años el Dr. Wall presenta unos severos dolores abdominales que culminan en el diagnóstico de una hernia inguinal estrangulada que requirió múltiples tratamientos. Sus recuerdos alrededor de esta enfermedad fueron muy traumáticos por el componente doloroso y la falta de medidas eficaces de control de los síntomas. Este sufrimiento durante un periodo de vulnerabilidad influyó en su posterior interés por el entendimiento y control del dolor. Inicio sus estudios en la Escuela San Pablo de Londres, la cual es una escuela privada de la ciudad capital fundada en 1509 por el diácono de la Catedral de Londres y uno de los más grandes pioneros de la educación llamado John Colet, quien fue un sacerdote inglés muy reconocido por sus comentarios bíblicos novedosos y por el desarrollo de nuevos métodos de educación. Cuando estaba en la escuela estalla la Segunda Guerra Mundial que trastorna las rutinas escolares y cambia radicalmente el modo de vida. Los niños fueron evacuados a unas 20 millas de Londres, en el oeste, y repartidos en diferentes hogares de la zona. Igualmente muchos de sus profesores tradicionales tuvieron que alistarse en las filas del ejército inglés e ir a la guerra. De esos años de colegio tiene un especial recuerdo por su profesor S. A. Barnett quien por problemas de salud no pudo ir a la guerra y recién graduado de zoología en la Universidad de Oxford fue nombrado director académico de la escuela. Desconocía todas las técnicas de enseñanza lo que permitió la ausencia de reglas y la posibilidad de que muchos del grupo del Dr. Wall desarrollaran sus ideas socialistas. Al terminar sus primeros estudios ingresó en la Universidad de Oxford que es el centro educativo universitario 
de habla inglesa más antiguo del mundo (1096), fundado cuando Enrique II de Inglaterra prohibió a los estudiantes ingleses la asistencia a los colegios de estudios superiores de Paris. En esta Universidad no solo se graduó como médico Patrick Wall sino también otros científicos reconocidos como William Harvey (describió la circulación sanguínea), Thomas Willis (neuroanatomista que describió el polígono de Willis) y Christopher Wren (anatomista). Fue durante mucho tiempo profesor de esta facultad Sir Charles Scott Sherrington, fisiólogo que estudió medicina en la Universidad de Cambridge pero que dedicó gran parte de su vida a la investigación en los laboratorios de Oxford. Sherrington recibió el Premio Nobel de Medicina en 1932 por sus trabajos en la localización de las funciones en la corteza cerebral, sus estudios en médula espinal y la actividad refleja. La parte clínica fue enseñada durante mucho tiempo en la Universidad de Oxford por Sir William Osler, quien era canadiense, hizo sus estudios en la Universidad de Mc Gill, trabajó en la Universidad de Pensilvania y fue profesor de medicina del Johns Hopkins. Desde 1905 llegó a Londres y se desempeñó en la Universidad de Oxford. Coleccionó libros de historia de la medicina, sus descripciones clínicas abundan y hay muchos signos y síndromes que llevan su nombre. La Universidad de Oxford acogió igualmente la historia de la penicilina. Primero Alexander Fleming quien cultiva unos hongos (penicillium notatum) en Agar y descubre como alrededor de dicha zona hay una ausencia total de bacterias. Le da el nombre de penicilina a la sustancia que impide el crecimiento de las bacterias pero no logra identificarla. Fue Essor Howard Florey, 10 años después del descubrimiento de Fleming, quien desarrolla una forma purificada de penicilina y comienza a utilizarla en humanos. Más adelante, también en estos laboratorios, Sir Edward Abraham descubre la cefalosporina.

Las primeras ideas socialistas inculcadas por su profesor Barnett motivaron al Dr. Wall a convertirse en presidente del club socialista de Oxford, el cual posteriormente se trasformaría en el partido comunista. El resurgir de las ideas socialistas apareció porque durante sus primeros años en Oxford se implantó a nivel nacional el "Sistema Nacional de Salud" contra el cual estaba la mayoría de los médicos y estudiantes. El Dr. Wall obtuvo el apoyo de la Asociación Médica Británica para organizar a los estudiantes en apoyo al sentimiento de protesta ante esta nueva política. Dicho apoyo se vio reflejado en la creación de la revista "British Medical Journal Students", dedicada a recoger las ideas de inconformidad de los estudiantes y plasmarlas en sus artículos.

Realiza sus estudios de medicina de una manera sobresaliente en la facultad de la Universidad de Oxford. Durante su periodo inicial logró vincularse al laboratorio de Alexander Fleming para trabajar durante las vacaciones en actividades de investigación. Recordemos que en este laboratorio Fleming (1881-1955) había realizado su trabajo con el cultivo de hongos en agar. Su formación médica se vio acompañada rápidamente de grandes logros y publicó sus primeras investigaciones en revistas de gran interés científico. Cuando cumplió 21 años ya tenía tres publicaciones científicas en dos de las más importantes revistas internacionales (Brain y Nature). Durante este periodo el Dr. Wall señala la influencia de algunos de sus profesores como fue el Dr. Paul Glees (1909-1999), un anatomista proveniente de Alemania quien había aceptado trabajo en Oxford buscando proteger de las leyes nazis a su esposa que era judía. Esta pareja de esposos se caracterizaron por ser generosos, acogedores y amables sobre todo con un grupo de estudiantes que se convirtieron en el "Grupo de Glees". Este grupo reunía algunos estudiantes motivados por las tendencias de investigación de Glees y por la necesidad del entendimiento de las ciencias básicas. Los trabajos de Glees fueron sobresalientes, describió la tinción de plata de "Glees" que permite ver la degeneración neuronal. Este método histológico fue rápidamente reemplazado por el método de Nauta, que permite ver la degeneración neuronal de los axones. Patrick Wall se vinculó no solo afectivamente, con los esposos Glees, sino laboralmente trabajando ampliamente en el laboratorio del anatomista. Publicaron (Glees y Wall) en 1946 un trabajo en el que determinan los cambios degenerativos en el núcleo centromedial del tálamo y el núcleo subtalámico (8-10). En 1948 obtuvo el diploma de especialista en Neurología.

En los primeros años de su actividad laboral se dedicó a la atención de las víctimas de la segunda guerra mundial, tanto aquellos que vivieron el holocausto judío como refugiados extranjeros que llegaron a Londres. Luego, se desplazó a Estados Unidos en donde trabajo hasta 1950 como instructor en la Escuela de Medicina de la Universidad de Yale. En esos pocos años en Yale trabajó arduamente en el análisis de la utilidad de la lobotomía para el tratamiento de la depresión. Hay que recordar que se denomina lobotomía a la destrucción quirúrgica de las vías de conexión de los lóbulos cerebrales, diferenciándola de la lobectomía en la cual se realiza la resección de uno o varios de los lóbulos cerebrales. Este trabajo del Dr. Wall se pone en el contexto de la época, cuando las "lobotomías prefrontales" (leucotomías prefrontales) estaban siendo utilizadas para el tratamiento de la depresión. Vale la pena señalar la relevancia médica de este procedimiento médico en la época, ya que fue descrito por Egas Moniz y Almeidas de Lima en la Universidad de Lisboa en el año 1935. Moniz después de evaluar los resultados de lobectomía frontal bilateral realizada en chimpancés por Fulton y Jacobson (1933), presentó los primero casos realizados en humanos en el Congreso Internacional de Neurología $(1935)(11,12)$. Moniz recibió el premio nobel de medicina en 1949 por la utilidad terapéutica de la leucotomía prefrontal en algunas forma de psicosis. En Estados Unidos Walter Freeman se convirtió en el mayor impulsor de esta técnica (no era ciru- 
Rev. Soc. Esp. del Dolor, Vol. 20, N. o 4, Julio-Agosto 2013

jano) pero desarrolló la técnica del "picahielo" en donde con un instrumento casero y a través del borde interno de la órbita realizaba este procedimiento, aún sin anestesia. En pocos años se alcanzaron a realizar solo en Estados Unidos cerca de 50.000 procedimientos. El último de ellos se realizó legalmente en 1967. Freeman perdió su licencia cuando uno de sus pacientes falleció. Otro de los casos más conocidos de malos resultados de este procedimiento fue el de Rosmery Kennedy (hermana del presidente John F. Kennedy) quien fue sometida a este procedimiento y quedó con secuelas graves que requirieron de un manejo institucional hasta su muerte a los 60 años. Durante su periodo en Yale resalta el Dr. Wall su relación con John Fulton (1899-1960), quien en 1920 llegó a Oxford para vincularse al laboratorio de fisiología de Sherrington, en donde realizó investigaciones sobre la corteza motora. Dichos trabajos los realizó en conjunto con J. C. Eccles (neurofisiólogo australiano que ganó el Premio Nobel de Medicina en 1967 por sus trabajos sobre la sinapsis). Fulton fue llamado en 1930 a reconstruir el departamento de fisiología de la Universidad de Yale, llegando a convertirlo en la Meca de la investigación para neurólogos, fisiólogos y neurocirujanos. Igualmente fundó en 1938 el Journal of Neurophysiology y se encargó de recopilar en los primeros volúmenes los adelantos sobre la fisiología del sistema nervioso. Efectivamente en 1934 Fulton y Jacobsen operan 2 chimpancés que se llamaban Becky y Lucy, realizándoles una lobectomía frontal. La calma observada después de la cirugía en estos animales motivó a Moniz y al resto del mundo a querer desocupar los hospitales psiquiátricos de pacientes al realizarles dicha cirugía. En 1948 Fulton escribió: "quiero llamar a la prudencia ya que no hay datos fisiológicos básicos que sustenten el funcionamiento de este procedimiento en los seres humanos". Fulton, en conjunto con los directivos de Yale, desarrolló un plan para determinar los principios fisiológicos de dicho procedimiento "Proyecto lóbulo frontal de Yale” y determinar su utilidad y su riesgos. En este proyecto y en estas investigaciones fue invitado el Dr. Wall (13-15).

Durante este periodo también compartió con Alex Mauro, quien era físico e ingeniero eléctrico. Desarrolló junto a Mauro técnicas de estimulación cerebral en animales. Este trabajo fue publicado en 1950 y permitió junto a Sweet desarrollar las técnicas de estimulación trascutánea que fue la base para la estimulación de los cordones posteriores en el tratamiento del dolor crónico (16). La primera serie de pacientes tratados con estos dispositivos fue publicado por Sweet y Wall en 1967. Los trabajos realizados por el Dr. Wall en esta época sobre la fisiología de la médula espinal comenzaron a dimensionar su nombre en el contexto científico, no solo de Estados Unidos. Fue así como después del Congreso Internacional de Montreal en donde Wall presentó muchas de sus investigaciones fue convocado a la oficina de Wilder Penfield (1891-1976), quien era un neurocirujano canadiense que desarrolló la cirugía de la epilepsia y trabajó con Sherrington en la Universidad de Oxford. En dicha reunión estaban también los investigadores de la Universidad de Mc Gill ampliamente conocidos en al ámbito mundial como Eccles y Jasper. El Dr.Wall les explico sus análisis en los cuales básicamente describía la importancia de la existencia de un control presináptico a los impulsos en su proceso de trasmisión. Penfield de una manera cordial le señaló que estaba equivocado en sus análisis y que todo radicaba en las malas influencias científicas que había tenido en la Universidad de Yale e incluso en Oxford. Le recomendó dejar de lado esas teorías equivocadas y redireccionar sus estudios. La sorpresa para el Dr.Wall fue cuando 5 años después Eccles (quien estaba en esa recordada reunión) publicó la misma teoría como propia sin mencionar los estudios previos de Wall. (17-25).

Después de la Universidad Yale el Dr. Wall pasó a ser profesor asistente en la facultad de medicina de la Universidad de Chicago hasta 1953, cuando va como profesor asistente a la Universidad de Harvard. En 1957 ingresó como profesor asistente y luego profesor titular (1960) del Instituto de tecnología de Massachusetts (Massachusetts Institute of Technology). Su llegada a este sitio marcó un evento histórico para la ciencia médica, ya que conoce a Ronald Melzack y comienza a trabajar en los principios básicos para el entendimiento del dolor. En este sitio da inicio la historia de una relación estrecha entre Patrick Wall y el dolor. Esa historia nos llevará más adelante a decir que fue el Dr. Wall uno de los principales especialistas en el mundo que permitieron el desarrollo del conocimiento del dolor. El MIT (Massachusetts Institute of Technology) nos conduce a los inmensos logros académicos, gremiales y asistenciales no solo del Dr. Wall sino también de R. Melzack. Más de 300 artículos publicados relacionados con el tema del dolor (logros académicos), la creación de la primera asociación mundial de especialistas interesados en el tema del dolor (logros gremiales) y los principios para el desarrollo de procedimientos terapéuticos que aún controlan el dolor de muchos pacientes (logros asistenciales). El Dr. Wall trabajó con William Sweet (reconocido neurólogo americano) en el desarrollo de las técnicas de estimulación eléctrica transcutánea y de los sistemas de estimulación cordonal posterior. William Sweet, que era mayor que él, trabajó mucho tiempo en el Servicio de Neurocirugía del Hospital General de Massachusetts. El grupo de investigación de Wall adquirió un reconocido sitio en el campo científico internacional, pero sus inclinaciones políticas volvieron a motivar un cambio radical en su vida. Investigaciones de la CIA no pudieron aclarar sus inclinaciones ideológicas en una época de guerra fría y de paranoia comunista. La presión política y administrativa le obligó a abandonar todo lo que había desarrollado y partir del "MIT". En 1967 regresa a Londres e ingresa a trabajar en el Colegio de Londres, siendo nombrado profesor titular de 
anatomía. Ese cambio abrupto del lujo y la comodidad de Estados Unidos y del Instituto de Tecnología de Massachusetts le generó inquietud y algo de preocupación. Fue John Z. Young quien ayudó a que ese cambio radical fuera mejor tolerado. Young era zoólogo, anatomista y filósofo, siendo en 1946 el primer jefe de un Departamento de Anatomía en Gran Bretaña sin el título de médico. Young fue ampliamente conocido por ser el primero en identificar el axón gigante del calamar (era especialista en cefalópodos) que permitió realizar a Hodking y Huxley los estudios del potencial de acción con el que obtuvieron el premio nobel. Young pasó mucho tiempo de su vida en Nápoles (Italia), en donde desarrolló ampliamente su afición e interés por los cefalópodos. Su amplio conocimiento de estos animales le permitió diferenciar esa estructura grande del calamar que muchos consideraban ligamentaria y confirmar que se trataba del axón gigante. El Dr. Wall va a Israel y se vincula en 1972 con la Universidad Hebrea de Jerusalén. (26-28). Entre sus premios resalta: Miembro de la Sociedad Real de Médicos (1984), Doctorado Honorífico en la Universidad de Siena (1987), Medalla Sherrington de la Sociedad Real de Medicina (1988), Miembro de la Real Sociedad (1989). Fue postulado durante varios años al Premio Nobel de Medicina, Miembro de la Real Sociedad de Anestesiólogos (1992), Doctorado Honorifico en la Universidad de Debrecen (1993), Medalla Real del Reino Unido (1999), Medalla de Honor de la Asociación Internacional para el Estudio del Dolor (IASP-1999).

Fue conocido con el sobrenombre de "The Pat" o la pared. Era fumador y reconocía que sus ideas lo ubicaban con tendencias de izquierda. Le gustaba observar las aves y disfrutaba de paseos para buscar especies raras. Se casó tres veces. Su primer matrimonio fue el 10 de agosto de 1950 con Betty Tucker (artista y poeta) se divorció en 1973. El segundo matrimonio fue el 26 de agosto de 1976 con Vera Ronnen (artista de Jerusalén) y finalmente Mary McLellan el 6 de mayo de 1999, dos años antes de su muerte. Después de su muerte su cuerpo fue donado al laboratorio de anatomía para ser usado en disección educativa.

Estando de vacaciones en Cork (segunda ciudad en importancia de la República de Irlanda) sufrió un desmayo por lo que debió ser atendido en el hospital y se le hizo en 1996 el diagnostico de un cáncer de próstata. Recibió tratamiento y presentó una remisión de 5 años. En el 2001 tuvo una recaída tumoral que necesita de una cirugía renal el 2 de agosto. Alcanza a recuperarse y salir a su hogar, pero el 8 de agosto fallece. Ese mismo año fallece William Sweet con quien había desarrollado las técnicas de electroestimulación.

\section{RONALD MELZACK}

Ronald Melzack nació en Montreal (en la zona judía) el 19 de julio de 1929. Montreal es la ciudad más grande de la provincia de Quebec y la segunda ciudad francófona más poblada en el mundo. Montreal es la ciudad más "educada" del mundo y aquella que tiene la mayor población de estudiantes universitarios per cápita en toda América del Norte. Nació en una familia de origen humilde y tuvo dos hermanos llamados Jack y Louis. La familia obtenía su sustento atendiendo una librería por lo que las limitaciones económicas eran importantes. Los padres de Melzack evaluaron que muy difícilmente iban a poder darle educación universitaria a los tres jóvenes por lo cual decidieron que solo uno de ellos podía completar su educación. Las cualidades de Ronald Melzack durante su periodo de colegio hicieron que fácilmente sus padres lo escogieran a él para ir a la universidad. Una vez Ronald pudo apoyar la actividad económica de la familia pudieron adquirir la librería y convertirla en una de las más importantes, llamada "Classic Books". Desde sus inicios mostró grandes habilidades académicas sin un especial interés hacia las ciencias puras. Fue la enfermedad sufrida por dos de sus hermanos, al parecer de origen tumoral, las que comenzaron a despertar en Melzack el interés por el dolor y el sufrimiento.

$\mathrm{Al}$ terminar el colegio decidió ingresar en la Universidad de Mc Gill para iniciar sus estudios en psicología. Desde esos primeros años el poder conocer y trabajar con Donald O. Hebb le permitió reforzar su interés en el conocimiento de la psicología humana. Hebb que nació en Chester (provincia de Nueva Escocia -una de las 10 provincias de Canadá) en 1904, es considerado el padre de la biopsicología. Desarrolló ampliamente el entendimiento de los principios fisiológicos y bioquímicos del comportamiento psicológico. Se dio a conocer relacionando la percepción, las emociones, el pensamiento y la memoria con las funciones fisiológicas de la actividad cerebral. Integró las sensaciones emocionales básicas con las estructuras cerebrales y buscó una explicación biológica, estructural, para cada uno de dichos procesos. Conoció a Pavlov de quien aprendió sus técnicas de experimentación animal, lo que le permitió probar sus teorías biopsicológicas. Hebb es también muy conocido en neurofisiología básica por la "ley de Hebb" que consiste en que "Cuando el axón de una célula A está lo suficientemente cerca de una célula B como para excitarla y participar continua y repetidamente de su disparo, ocurre algún proceso de crecimiento o de cambios metabólicos, en una o ambas células, de modo tal que aumenta tanto la eficiencia de A como la de una de las distintas células que disparan a B". El contacto de Ronald Melzack con Donald Hebb desde los inicios de su formación como psicólogo va a direccionar sus líneas de trabajo y hasta el desarrollo de su conocimiento a partir de una base biopsicológica amplia. Desarrolló el conductismo (estudio de la conducta humana) y se interesó de gran manera en el estudio de los miedos irracionales como por ejemplo el miedo de ciertas personas a abrir un paraguas. 
Rev. Soc. Esp. del Dolor, Vol. 20, N. o 4, Julio-Agosto 2013

Dentro de ese conductismo que tanto le apasionaba no podía caber mejor el estudio del dolor. Los patrones específicos de comportamiento en los pacientes con dolor, las conductas emocionales que envuelven el dolor, la vulnerabilidad dolorosa, la memoria del dolor, y otros temas fueron poco a poco llenando sus espacios y generando ideas de análisis y estudio. Realizó su doctorado en ciencias psicológicas en la Universidad de Mc Gill, asesorado por Hebb y dedicando su actividad investigativa al estudio del paciente con dolor de miembro fantasma. Esta motivación floreció después de conocer a una mujer que había perdido una pierna y presentaba una algohalucinosis (dolor de miembro fantasma). De forma paralela inició una actividad bastante dispendiosa que iba a verse representado en un importante desarrollo para la evaluación del dolor. Comenzó a recoger los adjetivos con los cuales los pacientes describían su manifestación dolorosa, reagrupándolos en la escala de evaluación cuantitativa del dolor, mejor conocido como "Escala de Mc Gill". Son preguntas de escogimiento múltiple que le permiten al paciente seleccionar los adjetivos que mejor representan su sintomatología. Otra de sus áreas de interés, en las cuales continuó trabajando, fue el miedo irracional al cual también le dedicó gran parte de su actividad. El doctorado que inicio en la Universidad de Mc Gill lo continúo en la Universidad de Oregón y posteriormente en la Universidad de Pisa en Italia. Tuvo la oportunidad de trabajar con el Dr. Livingston, quien en su laboratorio le permitió desarrollar múltiples modelos experimentales en ratas durante un periodo de 5 años.

En 1959 llega al Instituto de Tecnología de Massachusets en donde conoció a Patrick Wall y pudo desarrollar sus innovadores conceptos sobre la fisiopatología del dolor. Fue en 1965 cuando publica su "teoría de la compuerta" a la par del desarrollo en el conocimiento de las endorfinas, encefalinas y de las vías opioidérgicas. De forma paralela a sus trabajos con Wall desarrolló un protocolo de investigación sobre la analgesia inducida por el estrés, el dolor de miembro fantasma y la teoría de la neuromatriz publicada años después, como el paso siguiente a la "teoría de la compuerta". En 1968 publica en conjunto con Kenneth Cassey su trabajo relacionado con el origen del dolor, indicando que no es en la periferia, sino que el estímulo inicial parte de la periferia pero su interpretación es el resultado final de un complejo proceso de evaluación de datos, actuales, pasados y futuros integrados en una red neuronal distribuida por distintas zonas cerebrales. Inclusive se atreven a decir que el estímulo periférico inicial puede ni siquiera llegar a la corteza y la sensación de percepción dolorosa parte espontáneamente del cerebro a partir de un proceso evaluativo que incluye información de la lesión actual y se manifiesta como una reacción de alerta. Resaltan el concepto de "memoria del dolor" o memoria de predicción en la que de acuerdo a las características de la situación dolorosa el cerebro puede anticipar el síntoma en una actitud predictiva. Esa predicción puede anticipar incluso el momento en el que suceda algo en la zona donde se refiere la sensación. Estos conceptos se consolidan en 1989 cuando realiza la primera publicación en la que se menciona la neuromatriz como esa estructura básica sobre la cual se desarrolla la mayoría de las funciones neurológicas, incluyendo el dolor. Esa neuromatriz se considera como la estructura central responsable de la manifestación dolorosa. Completa este interesante análisis señalando que el dolor no es solo una sensación orgánica somatosensorial sino que incluye el aspecto emocional relacionado con el tono afectivo del sufrimiento, la conducta y la evaluación cognitiva del dolor que integra los aspectos sensoriales, emocionales presentes, antiguos y la experiencia dolorosa (memoria del dolor). En 1974 junto a Joseph Starford fundó la primera Clínica de Dolor en Canadá, denominada "Mc Gill Montreal Hospital Pain Centre". Melzack fue el director científico durante 26 años. En 1975 publicó el cuestionario de Mc Gill, ampliamente conocido en todo el mundo, traducido y validado en 20 idiomas. Todos los años de trabajo en el conocimiento del dolor se vieron recompensados en 1984 cuando fue nombrado Presidente de la Asociación Internacional para el Estudio del Dolor (IASP).

Melzack es un amplio conocedor de las tribus Inuit que son los habitantes de la región ártica. El término Inuit es la denominación común para los distintos pueblos esquimales que habitan las regiones árticas de América y Groenlandia. Inuit significa "pueblo" y el término en singular para denominar al "hombre" es Inuk. Estos términos han desplazado los términos originales que ya en esa zona no se utilizan. Inut reemplazó el término esquimal. Ronald Melzack publicó en 1977 el libro titulado "Why the man in the moon is happy and other eskimo creation stories retold by Ronald Melzach". Este libro fue premiado en su momento como la mejor obra literaria de Canadá. Entre los premios y reconocimientos obtenidos resalto: Premio D. O. Hebb de la Asociación Canadiense de Psicología (1986), Presidente de la Asociación Internacional para el Estudio del Dolor (1984-1987), Presidente de Honor de la Asociación Canadiense de Psicología (1988-1989), Premio Molson del Consejo Canadiense de literatura (Historias de Esquimales) (1985), doctorado de honor de la Universidad de Waterloo y de la Universidad de Dalhousie (1986), Premio de Ciencias Puras y Aplicadas de la Academia de Quebec (1994), Orden de Canada (1994), Orden de Quebec (2000), Premio Gravemeyer de Psicología. Son 5 premios dados anualmente por la Universidad de Louisville en el Estado de Kentucky en Estados Unidos (2010), Premio Killiam, incluido en el salón de la fama de la Asociación Médica Canadiense por contribución sobresaliente a la ciencia médica y mejoría de la salud y el bienestar de la población del mundo (2009). Profesor Emérito de la Universidad de Mc Gill (2010). 


\section{ANÁLISIS DEL ARTÍCULO}

Pain Mechanism: A New Theory. A gate control system modulates sensory input from the skin before it evokes pain perception and reponse. Science. 19 November 1965, Volume 150, Number 3699. (Fig. 1)

Es un artículo publicado en la revista Science por el Dr. Melzach identificado como profesor asociado en el Departamento de Psicología de la Universidad de Mc Gill (Montreal-Canada) y el Dr. Wall profesor del Departamento de Biología del Instituto de Tecnología de Massachussets (Cambridge-Estados Unidos). Tiene 9 páginas que van desde la 971 hasta la 979. Tiene 5 divisiones, incluyendo las referencias y las anotaciones. Son 4 graficas que acompañan al desarrollo del tema e ilustran de forma clara el planteamiento. Este esquema de escritura permite que el tema central sea la "Teoría de la Compuerta" pero precedido de las teorías que permitieron llegar al concepto expuesto. Solo dos teorías previas (teoría de especificidad y teoría por patrones) acompañadas de una figura de Descartes y una de los procedimientos quirúrgicos realizados durante el Siglo xx que apoya la teoría por patrones. La teoría de la compuerta se expone con una figura histológica y anatómica que da apoyo al concepto y con el esquema básico desarrollado por Melzack y Wall que explica claramente el principio. Fueron 78 referencias bibliográficas entre las cuales 13 pertenecen a alguno los autores ( 5 de Melzack, 7 de Wall y 1 en el que están ambos).

\section{Proceso}

Desde 1957 cuando llego al MIT Patrick D. Wall y luego cuando en 1959 llega Ronald Melzach, comenzó a gestarse el desarrollo de la "teoría de la compuerta". Ambos conocedores de los principios básicos del entendimiento del dolor se unieron para desarrollar la teoría. Hasta ese momento eran dos las teorías que dominaban el entendimiento del dolor.

\section{Teoría de especificidad}

Beecher, Pavlov, Hunt, Mc Intyre, Douglas, Ritchie, Poggio, Mountcastle, Casey. Cuando la piel es expuesta a un objeto punzante se desencadena un estímulo e inmediatamente aparece el dolor $(29,30)$. Esta teoría plantea que la conducción del dolor se realiza a través de una vía específica y exclusiva que se inicia en receptores perifericos de dolor y se continúa a través de fibras y vías ascendentes que llevan directamente el dolor a la corteza somatosensorial. Se ilustra dicha teoría con el dibujo clásico de Descartes que aparece en su libro "l'homme" publicado en Paris en 1644. Esta figura se encuadra bajo el concepto de la "Teoría

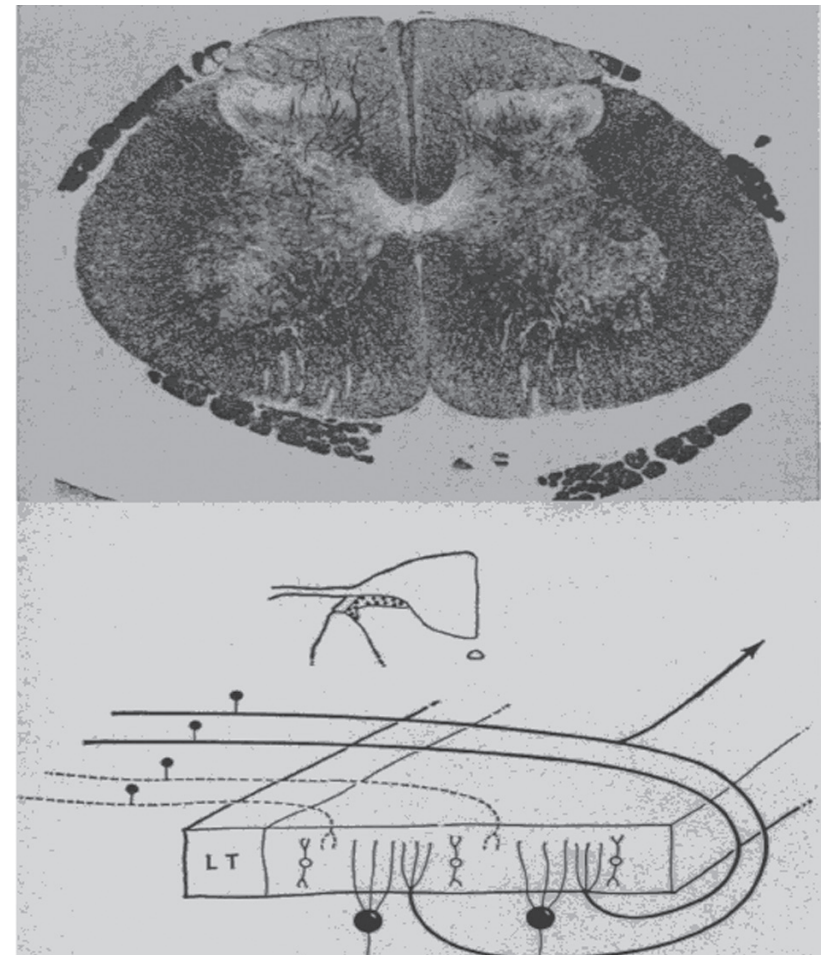

Fig. 1. Esquema utilizado por Melzach y Wall en el que se ilustra las vías anatómicas de conducción del dolor y en el cual se describen los diferentes procedimientos neuroquirúrgicos que se pueden realizar. Utilizan este esquema para debatir tanto la teoría específica como la teoría de los patrones. Si la vía del dolor fuera una sola que funcionara solo como un conducto que lleva el estímulo de la periferia a la corteza (teoría específica) la sección quirúrgica de la vía del dolor serviría para controlar todos los dolores. Por el contrario si el dolor dependiera exclusivamente del patrón de estimulación que reciben los receptores (teoría de patrones) la cirugía del dolor nunca serviría ya que no modifica esos aspectos cualitativos del dolor.

Cartesiana" que perduró como válida hasta finales del siglo XIX. El planteamiento de Descartes que va de la mano con la teoría específica, establece que los nervios (inclusive los relacionados con el dolor) son como una cuerda extendida y a tensión que va desde la periferia hasta la corteza. Si dicha cuerda se hace vibrar en uno de sus extremos, la vibración se desplaza a lo largo de la cuerda hasta llegar a su otro extremo en donde se extingue el estímulo. De la misma forma se explica la conducción de los estímulos al señalar que se inician en el extremo periférico de los nervios y se desplaza a través de los nervios "como si fuera una cuerda extendida" hasta la corteza somatosensorial específica. Max Von Frei planteó entre 1894 y 1896 las bases a la teoría específica, señalando que los receptores descubiertos de tejido conectivo (terminaciones libres) eran específicos de la vía de conducción del dolor y era la puerta 
Rev. Soc. Esp. del Dolor, Vol. 20, N. o 4, Julio-Agosto 2013

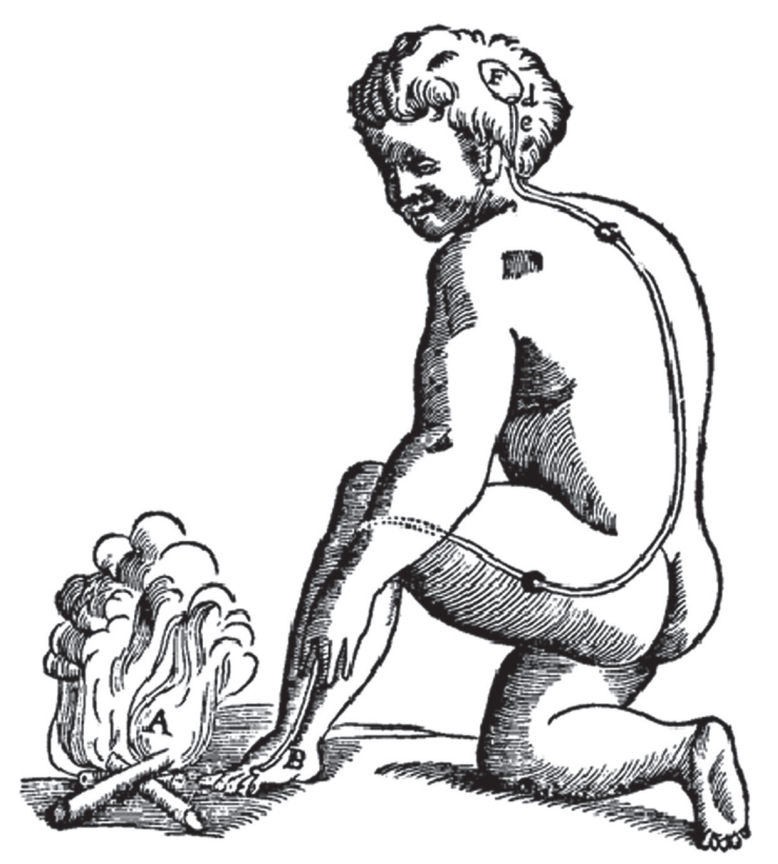

Fig. 2. Teoría Cartesiana de la conducción del sistema nervioso. Esta teoría fue planteada por René Descartes en su libro "L'homme". Melzach y Wall la utilizan en su artículo para ilustrar los conceptos de la teoría específica en la cual se considera que la conducción del dolor se realiza a través de vías específicas y exclusivas para la nocicepción, tal como lo ilustraba Descartes en el siglo XVII.

de entrada para aquellos estímulos dolorosos (31,32). De la misma manera se describieron los corpúsculos de Ruffini específicos para la sensibilidad al calor y los de Kraus para el frio. En el artículo se desvirtúa esta teoría a partir de las siguientes evidencias:

- Evidencia clínica: utilizan como ejemplo tres patologías dolorosas como son la causalgia (hoy llamado síndrome doloroso regional complejo), el dolor de miembro fantasma y las neuralgias periféricas. A partir de estas tres señalan:

- En estos pacientes la sección quirúrgica de una fibra nerviosa no suprime el dolor. Si existiera la "cuerda" basta solo con cortarla para interrumpir el estímulo y suprimir el dolor. En ninguna de estas tres patologías sucede esto (33).

- En estos pacientes la estimulación táctil (no dolorosa) de la piel en la zona del dolor es suficiente para generar un terrible dolor. Este fenómeno es conocido actualmente como alodinia (34).

- En estos pacientes se puede incluso ver dolor de aparición espontánea aun sin la presencia de ningún estímulo. El dolor aparece aun sin poner a vibrar la cuerda extendida, es decir aun sin producirse ningún estímulo. Estos fenómenos se explican actual-
19 November 1965, Volume 150, Number 3699

\section{SCIENCE}

\section{Pain Mechanisms: A New Theory}

A gate control system modulates sensory input from the skin before it evokes pain perception and response.

Fig. 3. Artículo original de la teoría de la compuerta.

mente por la presencia de una actividad ectópica espontánea a lo largo de las vías de conducción del dolor $(35,36)$.

- Lejos de la zona del dolor se pueden encontrar áreas de sensibilidad aumentada y puntos gatillo que activan el dolor. Lo que va en contra de una vía única de conducción asociada con cada receptor no encapsulado (terminación libre) $(37,38)$.

- Los pacientes que presentan hiperpatía siguen sintiendo el dolor aun cuando el estímulo se ha retirado del área afectada, también conocido este signo clínico como sumación temporal. Si la teoría específica fuera real inmediatamente al estímulo se suspende el dolor también. Esto no sucede en los pacientes con hiperpatia $(39,40)$.

- Evidencia psicológica. Algunos ejemplos de comportamientos psicológicos particulares, especialmente aquellos que se ven acompañados de situaciones de estrés, pueden demostrar como la teoría específica desconoce completamente la esfera psicológica.

- Cuando un soldado se encuentra en el campo de batalla y sufre cualquier tipo de herida puede incluso seguir luchando sin sentir ningún tipo de dolor. Por el contrario si la persona tiene la misma lesión en otras circunstancias puede ser sentida como severamente intensa (41).

- Pavlov realizó modelos experimentales en los que al mismo tiempo que a los animales se les presentaba la comida se les aplicaba un estímulo doloroso. Posteriormente Pavlov podía evocar la sensación dolorosa en los animales y generar un comportamiento doloroso solo con exponer al animal a la comida sin ningún estímulo doloroso $(42,43)$.

- Evidencia fisiológica: aunque se acepta que sí hay receptores específicos para el dolor estos son solo un pequeño porcentaje de todos los receptores que participan en la conducción del dolor. Aunque en el SNC existan zonas especializadas con neuronas "de dolor", lo que más frecuentemente se observa es la presencia, en la médula y a lo largo de las vías ascendentes, de neuronas de "amplio rango" que responden no solo 


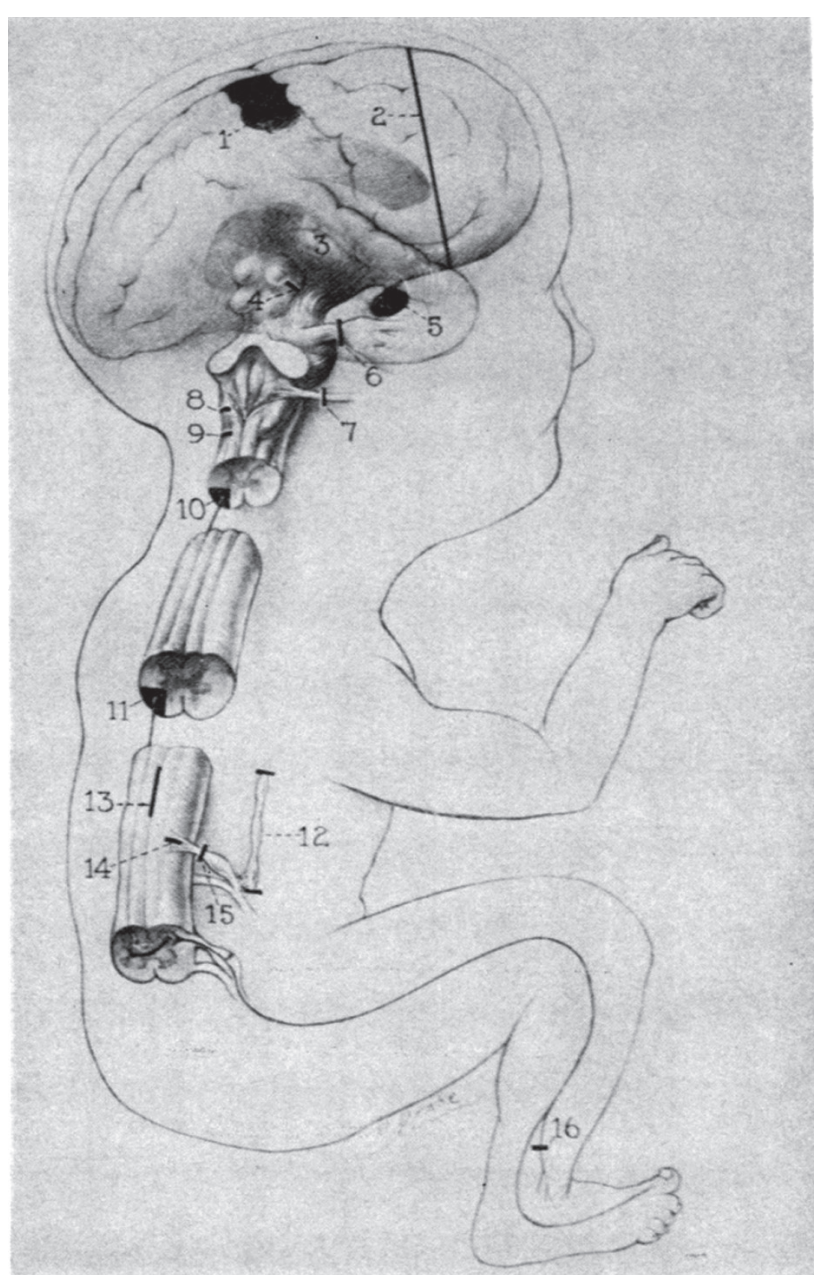

Fig. 4. Arriba: corte de médula espinal en gato. En el medio: esquema de cuadrante posterior derecho de la médula en donde se observa punteado la sustancia gelatinosa de Rolando. Abajo: el corte trasversal de la sustancia gelatinosa de Rolando en donde la líneas gruesas representan la sensibilidad cutánea (profunda), la cual termina una parte en la sustancia gelatinosa de Rolando y la otra en los cordones posteriores de la médula. Las líneas interrumpidas representan las fibras de pequeño calibre que llegan directamente a la sustancia gelatinosa de Rolando. Las esferas negras grandes son las neuronas que envían directamente sus dendritas a la sustancia gelatinosa de Rolando y son las que están relacionadas con las fibras de grueso calibre. Sus respectivos axones se comunican con el cuerno posterior de la médula. Los círculos blancos con las neuronas de la sustancia gelatinosa de Rolando y sus axones comunican con el tracto de lissauer. Este esquema da el soporte histológico y funcional para la "teoría de la compuerta".

al dolor. Fisiológicamente sí hay estructuras específicas para el estímulo doloroso pero no son las únicas que participan en el momento de conducir un dolor, incluso corresponden a un porcentaje mucho menor que otras. De esta forma así como en la médula hay

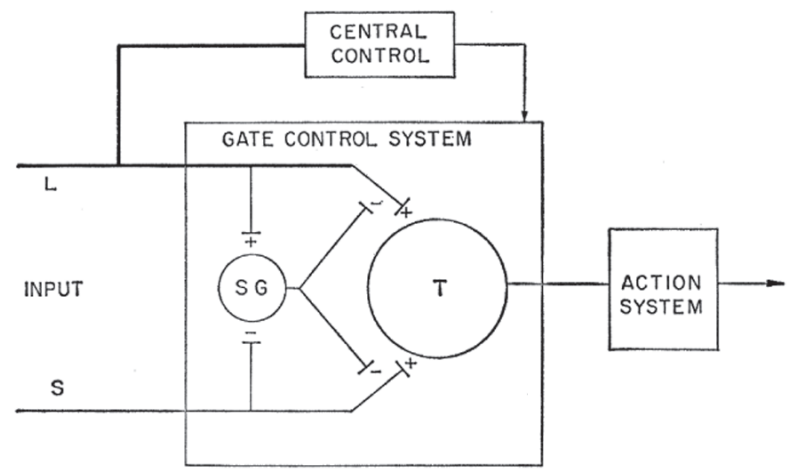

Fig. 5. Esquema original de la "teoría de la compuerta". (L) fibras de grueso calibre. (S) fibras de pequeño calibre. (SG) Sustancia gelatinosa de Rolando. (T) Neuronas centrales de trasmisión. Tanto las fibras de grueso calibre (L) como las de pequeño calibre (S) llegan a la sustancia gelatinosa de Rolando (SG) ejerciendo efectos opuestos. Las L-excitario sobre la SG es decir inhibitorio sobre el dolor y las $\mathrm{S}$ inhibitorios sobre la SG es decir excitatorio sobre el dolor. La sustancia gelatinosa de Rolando ejerce un efecto inhibidor sobre las células $\mathrm{T}$ pero depende ese efecto de la acción directa de las fibras de grueso o de pequeño calibre.

neuronas de "amplio rango" que responden a gran variedad de estímulos la participación de otras fibras "no específicas" es alta $(44,45)$.

\section{Teoría de patrones o de codificación de la actividad neuronal de Sherrington}

Esta teoría fue postulada por Sherrington en 1900 y basada en la ausencia de especificidad para los receptores del dolor. Esta teoría considera que la sensación dolorosa se explica por la intensidad del estímulo y no por la calidad del estímulo. Es decir, el dolor es un tipo de estímulo lo suficientemente intenso para generar una sensación dolorosa. El estímulo es doloroso por su intensidad y no por su cualidad. Esa hipótesis de Sherrington se apoyó en observaciones clínicas y solo años después se plantearon modelos experimentales para tratar de apoyarla. Alfred Goldschire, que era un seguidor de las teorías de Frei, posteriormente determinó como el dolor se produce no solo por la intensidad del estímulo sino también por la sumación de los impulsos a nivel central. Se apoyó en dos evidencias clínicas para ampliar esta teoría (46-49).

- Cuando se le aplica un estimulo térmico utilizando intenso calor a un paciente que sufre de "Tabes", va a sentir inicialmente un estímulo térmico que a medida que pasan los minutos se torna terriblemente doloroso. Explica dicha evidencia a partir de la sumación presente a nivel medular que explica la aparición del dolor. 
Rev. Soc. Esp. del Dolor, Vol. 20, N. o 4, Julio-Agosto 2013

- La estimulación de la piel con la cabeza de un alfiler inicialmente es bien tolerada pero después de un cierto tiempo se convierte en dolorosa.

Goldschire establece que lo que produce el dolor es la sumación de estímulos y la convergencia de sensaciones que se presenta en el cuerno posterior de la médula. Weddel y Sinclair desarrollan en 1955 la forma más simple de la teoría de patrones, denominada teoría de patrón periférico (50-52). Ellos consideran que el dolor está provocado por un exceso de estimulación periférica que produce un patrón de impulsos nerviosos interpretados centralmente como dolor. Son los patrones espaciales y temporales de impulsos nerviosos los que controlan la conducción del dolor más que las rutas separadas de trasmisión específica de cada modalidad $(53,54)$.

La teoría de patrones comenzó a ser debatida por Livingston cuando propuso el concepto de sumatoria central, en la que señala que la estimulación patológica de los nervios sensitivos activa circuitos reverberantes de las neuronas medulares, generando y potencializando el estímulo doloroso. Esta actividad anormal puede ser disparada por estímulos no dolorosos y de esa forma generar una descarga de impulsos nerviosos que son interpretados centralmente como dolor $(55,56)$.

\section{Análisis}

La percepción, conducción e interpretación del dolor no es solamente el desplazamiento de un potencial de acción a través de un circuito estático. El proceso del dolor implica la participación del sistema nervioso periférico y central en un proceso de modulación de ese estímulo doloroso. Las tres neuronas de la vía de conducción del dolor no son solamente relevos que permiten conducir el estímulo hasta la corteza, son neuronas que interactúan con un sistema periférico y central de control del estímulo doloroso. Ese sistema de control implica no solamente aspectos puramente sensoriales sino también aspectos afectivos y evaluativos. Se incorporan aspectos biológicos y psicológicos en la conformación del dolor siendo la percepción del dolor un proceso multidimensional. La teoría está centrada en la presencia de una "compuerta" en el asta dorsal de la medula. Esta compuerta por donde pasa el estímulo doloroso, se ve influenciada por la activación de fibras A-beta, las cuales son fibras de grueso calibre mielinizadas que inhiben la trasmisión (cierran la compuerta) y la conducción de las fibras A-delta y C (encargadas de conducir los estimulos dolorosos abriendo la compuerta). Las fibras mielinizadas de grueso calibre activan a la vez mecanismos supratentoriales de control del dolor y del control de la compuerta, que actúan a través de las vías inhibitorias descendentes (57-59).

\section{Propuesta}

Cuando se realiza un estímulo en la piel se activan dos tipos de fibras. Las primeras son las fibras de pequeño calibre amielínicas o poco mielinizadas que conducen los estímulos dolorosos, térmicos y táctiles superficiales. Las segundas son las fibras de grueso calibre mielinizadas que conducen estímulos propioceptivos como la presión, vibración o tacto profundo, entre otros. Las fibras de pequeño calibre llegan a través de la fibra aferente primaria al cuerno posterior de la médula. Las fibras de grueso calibre llegan también por la fibra aferente primaria a los cordones posteriores. Estas últimas tienen vías de conexión con las fibras de pequeño calibre en el cuerno posterior, a través de la rama colateral recurrente de Cajal. En el cuerno posterior existen las células "neuronales" $\mathrm{T}$ que son las que van a determinar el estímulo doloroso que será trasmitido a la corteza somatosensorial (60-62). Estas células T tiene una dependencia directa de las neuronas de la sustancia gelatinosa de Rolando, que ejerce el papel de "compuerta" o regulador de la información trasmitida a las células $\mathrm{T}$ y por consiguiente a la corteza somatosensorial. Las fibras de pequeño calibre ejercen un efecto inhibidor sobre la sustancia gelatinosa de Rolando, lo que permite que dicha sustancia no ejerza su efecto inhibidor sobre las células $\mathrm{T}$ y el estímulo doloroso se trasmita intensamente a las estructuras supratentoriales $(63,64)$. Por su parte las fibras de grueso calibre ejercen un efecto activador sobre las células de la sustancia gelatinosa de Rolando, favoreciendo el efecto inhibidor de las neuronas de la sustancia gelatinosa de Rolando sobre las células $\mathrm{T}$ y bloqueando el estímulo doloroso $(65,66)$. Cuando se produce una estimulación fuerte y prolongada, es decir de tipo nociceptivo, activa a la vez fibras de grueso y de pequeño calibre, resultando en un conflicto entre la acción de las primeras que tienden a cerrar la compuerta y las segundas que tienden a abrirla. Las de grueso calibre al tener una mayor velocidad se adaptan rápido, permitiendo que sean las de pequeño calibre las que finalmente abran la compuerta y permitan que el dolor pase (67-69).

\section{Las ilustraciones originales}

- Figura N1. Teoría cartesiana de conducción de los estímulos planteada por Descartes en su libro "l'homme" y tomado de la edición publicada en París en 1644. En ella se observa un niño arrodillado poniendo su pie izquierdo en contacto con una llama ardiente. Se ilustra la vía específica de conducción del estímulo desde la periferia hasta la corteza como una cuerda estirada (Fig. 2).

- Figura N2. Es una figura tomada del artículo de Mac Carty y Drake publicado en 1956 y correspondiente 
con el manual de procedimientos de la Clínica Mayo. En ella se ilustra el cuerpo humano, un poco deforme que pareciera corresponder a un niño en el que se ve el sistema nervioso central y periférico. El objetivo inicial de los autores para esta figura era ilustrar los diferentes procedimientos quirúrgicos de interrupción de vías para el tratamiento del dolor: girectomías, lobotomías prefrontales, talamotomías, tractotomías mesencefálicas, etc. Melzack y Wall usan este artículo para ilustrar uno de los argumentos con los que refutan las teorías específicas y de patrones. Cuando se secciona la vía del dolor no siempre se logra controlar el síntoma (refuta la teoría de la especificidad) pero en algunas casos sí se controla el síntoma (refuta la teoría de patrones) (Fig. 3).

- Figura N3. Esta es una figura creada por los autores. En la parte superior presentan la sección de la médula de un gato. En el medio presentan un esquema del sitio en el que van a realizar una sección trasversal del tejido y abajo un esquema del comportamiento de las fibras en la sustancia gelatinosa de Rolando y el cuerno posterior de la médula (Fig. 4).

- Figura N4. Teoría de la compuerta (Fig. 5).

\section{CORRESPONDENCIA:}

Juan Carlos Acevedo González

Departamento de Neurociencias

Hospital Universitario San Ignacio

Carrera 7 Numero 40-62

Bogotá, Colombia.

e-mail: jacevedog@gmail.com

\section{BIBLIOGRAFÍA}

1. Melzack R, Wall PD. On the nature of cutaneous sensory mechanisms. Brain. 1962;85:331-56.

2. Melzack R, Wall PD. Pain mechanisms: A new theory. Science. 1965;150:971-9.

3. Melzack R, Wall PD, Ty TC. Acute pain in the emergency clinic; Latency of onset and descriptor patterns related to different injuries. Pain. 1982;14:33-43.

4. Wall PD. The gate control theory of pain mechanisms. A reexamination and re-statement. Brain. 1978;101:1-18.

5. Wall PD. Do nerve impulses penetrate terminal arborizations? A pre-presynaptic control mechanism. Trends Neurosci. 1995;18:99-103.

6. Wall PD. Some unanswered questions about the mechanism and function of presynaptic inhibition. In: Rudomin P, Romo R, Mendell LM (editors). Presynaptic inhibition and presynaptic control. New York: Oxford University Press, 1998. p. 228-44.

7. Wall PD. Pain in context: The intellectual roots of pain research and therapy. In: Devor M, Rowbotham M, Wiesenfeld-Hallin Z (editors). Progress in pain research management. Seattle: IASP Press. 2000. Vol 17.
8. Glees P, Wall PD. Fibre connections in the subthalamic region and the contromedial nucleus of the thalamus. Brain. 1946;69:195-207.

9. Glees P, Wall PD. Commissural fibres of the macaque thalamus. An experimental study. Journal of Comparative Neurology. 1948;88:129-37.

10. Glees P, Wall PD, Wright TA. An ensheathed rotating knife for causing brain lesions. Nature. 1947;160:365.

11. Fulton JF, Pribram KH, Stevenson JAF, Wall PD. Interrelations between orbital gyrus, insula, temporal tip and anterior cingulated. Trans Am Neurol Assoc. 1949;175-9.

12. Wall PD, Davis GD. Three cerebral cortical systems affecting autonomic function. J Neurophysiol. 1951;14:507-18.

13. Wall PD, Glees P, Fulton JF. Corticofugal connections of posterior orbital surface on the rhesus monkey. Brain. 1951;74:66-71.

14. Wall PD, McCulloch WS, Lettvin JY, Pitts W. Terminal arborisations of the cat's pyramidal tract determined by a new technique. Yale J Biol. 1955;28:457-64.

15. Dubner R. A tribute to Patrick D. Wall. Pain. Suppl 1999;6:1156.

16. Wall PD, Sweet WH. Temporary abolition of pain in man. Science. 1967;155:108-9.

17. Wall PD. Excitability changes in afferent fibre terminations and their relation to slow potentials. L Physiol. 1958;143:121.

18. Wall PD. Cord cells responding to touch damage and temperature of skin. J Neurophysiol. 1960;23:197-210.

19. Wall PD. The origin of a spinal cord slow potential. J Physiol. 1962;164:508-26.

20. Wall PD. Presynaptic control of impulses at the first central synapse in the cutaneous pathway. Prog Brain Res. 1964;12;92-118.

21. Wall PD. The laminar organization of dorsal horn and effects of descending impulses J Physiol. 1967;188:403-23.

22. Wall PD, Gutnick M. Properties of afferent nerve impulses originating from a neuroma. Nature. 1974b;248:740-3.

23. Wall PD, Horwitz NH. Observations on the physiological action of strychnine. J Neurophysiol. 1951;14:257-63.

24. Wall PD, Johnson AR. Changes associated with post-tetanic potentiation of a monosynaptic reflex. J Neurophysiol. 1958;21:148-58.

25. Wall PD, Lidierth M. Five sources of a dorsal root potential: Origins and interactions J Neurophysiol. 1997;78:860-71.

26. Wall PD, Noordenbos W. Sensory functions which remain in man after complete transaction of dorsal horns. Brain. 1978;100:641-53

27. Wall PD, Werman R. The physiology and anatomy of long ranging afferent fibres within the spinal cord. J Physiol (London) 1976;255:321-334.

28. Wall PD, Woolf CJ. Muscle but not cutaneous C-afferent input produces prolonged increases in the excitability of the flexion reflex in the rat. J Physiol. 1984;356:443-58.

29. Heimer L, Wall PD. The dorsal root distribution of the substantial gelatinosa of the rat with a note on the distribution in the cat. Experimental Brain Research. 1968;6:89-99.

30. Howland B, Lettvin JY, McCulloch WC, Pitts W, Wall PD. Reflex inhibition by dorsal root interaction. J Neurophysiol. 1955;18:1-17.

31. Mauro A, Wall PD, Davey LM, Scher AM. Central nervous stimulation of implanted high frequency receiver. Fed Proc. 1950;9:86.

32. Basbaum AI, Wall PD. Chronic changes in the response of cells in adult cat dorsal horn following partial deafferenta- 
Rev. Soc. Esp. del Dolor, Vol. 20, N. o 4, Julio-Agosto 2013

tion; The apperance of responding cells in a previously nonresponsive region. Brain Res. 1976;116:181-204.

33. Wall PD, Cronly-Dillon JR. Pain, itch and vibration. Arch Neurol. 1960;2:365-75.

34. Devor M, Wall PD. Reorganisation of spinal cord sensory map after peripheral nerve injury. Nature. 1978;275:75-6.

35. Carlen PL, Wall PD, Nadvorna MD, Steinbach T. Pahantom limibs and related phenomena in recent traumatic amputations. Neurology. 1978;28:211-7.

36. Barbut T, Polak JM, Wall PD. Substance P in spinal cord dorsal horn decreases following peripheral nerve injury. Brain Res. 1981;205:289-98.

37. Allan JDB, Bultitude MI, Bultitudes MF, Wall PD, McMahon SB. The effect of capsaicin on renal pain signalling systems in humans and Wistar rats. J Physiol. 1997;505:39.

38. Devor M, Wall PD. The effect of peripheral nerve injury on receptive fields of cells in the cat spinal cord. J Comp Neurol. 1981a;199:277-91.

39. Devor M, Wall PD. Plasticity in the spinal cord sensory map following peripheral nerve injury in rats. J Neurosci. 1981b;1(7):679-84.

40. Dostrovsky J, Millar J, Wall PD. The inmediate shift afferent drive of dorsal column nucleus cells following deafferentation: A comparison of acute and chronic deafferentation in gracile nucleus and spinal cord. Exp Neurol. 1976;52:480-95.

41. McMahon SB, Wall PD. Plasticity in the nucleus gracilis of the rat. Exp Neurol. 1983b;80:195-207.

42. McMahon SB, Wall PD. Receptive fields of rat lamina 1 projection cells move to incorporate a nearby region of injury. Pain. 1984;19:235-47.

43. McMahon SB, Wall PD. Descending excitation and inhibition of spinal cord lamina 1 projection neurons. J Neurophysiol. 1988;59:1204-19.

44. Lidierth M, Wall PD. Synchronous inherent oscillations of potentials within the rat lumbar cord. Neurosci Lett. 1996; 220:25-8.

45. Lidierth M, Wall PD. Superficial dorsal horn cells connected to the Lissauer tract and their relation to the dorsal root potential. J Neurophysiol. 1997;80:667-79.

46. Noordenbos PD, Wall PD. Diverse sensory functions with an almost divided spinal cord. A case of spinal cord transection with preservation of part of one anterolateral quadrant. Pain. 1976;2:185-95.

47. Noordenbos W, Wall PD. Implications of the failure of nerve resection and graft to cure chronic pain produced by nerve lesions. J Neurol Neurosurg Psych. 1981;44:1068-73.

48. Wall PD, Devor M. The effect on peripheral nerve injury on dorsal root potentials and on transmission of afferent signals into the spinal cord. Brain Res. 1981;209:95-111.

49. Wall PD, Devor M. Sensory afferent impulses from dorsal root ganglia as well as from the periphery in normal and nerve injured rats. Pain. 1983;17:321-39.

50. Wall PD, Woolf CJ. The brief and the prolonged facilitatory effects of unmyelinated afferent input on the rat spinal cord are independently influecend by peripheral nerve injury. Neuroscience. 1986;17:1199-206.

51. Wall PD, Yaksh TL. Effect of Lissauer tract stimulation on activity in dorsal roots and in ventral roots. Exp Neurol. 1978;60:570-83.
52. Wall PD, Waxman S, Basbaum AI. Ongoing activity in peripherañ nerve: 111. Injury discharge. Exp Neurol. 1974; 45:576-89.

53. Wall PD, Fitzgerald M, Nussbaumer JC, Van Der Loos H, Devor M. Somatotopic maps are disorganized in adult rodents treated with capsaicin as neonates. Nature. 1982a; 295:691-3

54. Wall PD, Scadding JW, Tomkiewicz MM. The production and prevention of experimental anaesthesia dolorosa. Pain. 1979b;6:175-82.

55. Wall PD, Devor M, Inbal R, Scadding JW, Schonfeld D, Seltzer Z, Tomkiewicz MM. Autotomy following peripheral nerve lesions; Experimental anaesthesia dolorosa. Pain. 1979a; 7:103-13.

56. Wall PD, Fitzgerald M, Woolf CJ. Effects of capsaicin on receptive fields and on inhibitions in rat spinal cord. Exp Neurol. 1982b;78:425-36.

57. Egger MD, Wall PD. The plantar cushion reflex circuit: An oligosynaptic reflex. J Physiol. 1971;216:483-501.

58. El-Sobky A, Dostrovsky JO, Wall PD. Lack of effect of naloxone on pain perception in humans. Nature. 1976;263:7834.

59. Fitzgerald M, Wall PD, Geodert M, Emson PC. Nerve growth factor counteracts the neurophysiological and neurochemical effects of chronic sciatic nerve injury. Brain Res. 1985;332:131-41.

60. McMahon SB, Wall PD. A system of rat spinal cord lamina 1 cells projecting through the contralateral dorsolateral funiculus. J Comp Neurol. 1983a;214:217-23.

61. Nussbaumer JC, Wall PD. Expansion of receptive fields in the mouse cortical barrelfield after administration of capsaicin to neonates or local application on the infraorbital nerve in adults. Brain Res. 1985;360:1-9.

62. Scadding JW, Wall PD, Wynn Parry CB, Brooks Dm. Clinical trial of propranolol in post-traumatic neuralgia. Pain. 1982;14:283-92.

63. Thompson SWN, Wall PD. The effect of GABA and 5-HT antagonists on rat dorsal root potentials. Neurosci Lett. 1996;217:153-6.

64. Wall PD. The presence of ineffective synapses and the circumstances which unmask them. Philos Trans R Soc London. B 1977;278:361-72.

65. Wall PD, Fitzgerald M. Effects of capsaicin applied locally to adult peripheral nerve. 1. Physiology of peripheral nerve and spinal cord. Pain. 1981;11:363-77.

66. Wall PD, Gutnick M. Ongoing activity in peripheral nerves: 11. The physiology and pharmacology of impulses originating in a neuroma. Exp Neurol. 1947a;43:580-93.

67. Wall PD, Mills R, Fitzgerald M, Gibson SJ. Chronic blockade of sciatic nerve transmission by tetrodotoxin does not produce central changes in the dorsal horn of the spinal cord of the rat. Neurosci Lett. 1982c;30:315-20.

68. Wall PD, Lidierth M, Hillman P. Brief and prolonged effects of lissauer tract stimulation on dorsal horn cells. Pain. 1999;83:579-89.

69. Woolf CJ, Wall PD. The relative effectiveness of C-primary afferents of different origins in evoking a prolonged facilitation on the flexor reflex in the rat. J Neurosci. 1986;6:143342 . 